\title{
Some Results on Additive Number Theory IV
}

\author{
Minoru TANAKA \\ Gakushuin University
}

\section{§1. The main theorem.}

Let $\omega(n)$ denote the number of distinct prime factors of a positive integer $n$.

THEOREM. Let $\alpha<\beta$. Let $A(N ; \alpha, \beta)$ denote, for sufficiently large positive integer $N$, the number of representations of $N$ as the sum of the form $N=p+n$, where $p$ is prime, and $n$ is a positive integer such that

$$
\log \log N+\alpha \sqrt{\log \log N}<\omega(n)<\log \log N+\beta \sqrt{\log \log N},
$$

then, as $N \rightarrow \infty$, we have

$$
A(N ; \alpha, \beta) \sim \frac{N}{\log N} \cdot \frac{1}{\sqrt{2 \pi}} \int_{\alpha}^{\beta} e^{-x^{2} / 2} d x
$$

We shall give a proof of this theorem in section 2. Our proof runs in the same lines as in my paper [6], but it uses also Bombieri's mean value theorem and Brun-Titchmarsh's inequality. It is to be noticed that somewhat analogous theorem was proved in Halberstam [3] using SiegelWalfisz's theorem. It might perhaps be possible to prove our theorem in a similar style as in [3], but I hope that it would be of interest to prove the theorem in our way.

As was shown in Gallagher [2], Bombieri's theorem can be deduced rather simply from Siegel-Walfisz's theorem, and is far more conveniently applicable in our situation. For Bombieri's theorem cf. Bombieri [1], Gallagher [2], Halberstam-Richert [4], p. 111, Mitsui [5], Chap. 8.

We shall shorten the paper by omitting the similar parts of the proof as in Tanaka [6].

The author expresses his thanks to Prof. S. Iyanaga for his kind advices. 


\section{§2. Proof of the main theorem.}

Let $a$ and $b$ be non-negative integers. Then

$$
\sum_{o=0}^{b}(-1)^{\circ}\left(\begin{array}{l}
a \\
c
\end{array}\right)\left\{\begin{array}{lll}
=1, & \text { when } a=0, \\
\geqq 0, & \text { when } a>0 \text { and } b \text { is even, } \\
\leqq 0, & \text { when } a>0 \text { and } b \text { is odd. }
\end{array}\right.
$$

This is the same as Lemma 2 in [6].

Now we define some functions and sets which will be used in the sequel. The positive integer $N$ will be assumed to be sufficiently large as occasion demands.

We define the set $Q_{N}$ consisting of primes as

$$
\boldsymbol{Q}_{N}=\left\{p: p \nmid N, e^{(\log \log N)^{2}}<p<N^{(\log \log N)^{-2}}\right\}
$$

and put

$$
y(N)=\sum_{p \in Q_{N}} \frac{1}{p}
$$

Then we have

LEMMA 1. $y(N)=\log \log N+O(\log \log \log N)$.

Proof. We can easily see that $\omega(N)=O(\log N)$, and hence

$$
\sum_{p \mid N} \frac{1}{p} \leqq \sum_{p \leq \omega(N)} \frac{1}{p}=O(\log \log \log N) .
$$

The lemma can be obtained similarly as Lemma 4 in [6].

We denote by $\omega_{N}(n)$ the number of distinct prime factors of a positive integer $n$, which belong to the set $Q_{N}$ :

$$
\omega_{N}(n)=\sum_{p \mid n, p \in Q_{N}} 1 .
$$

For any positive integer $t$, we define the set $M_{N}(t)$ consisting of positive integers as

$$
\begin{aligned}
M_{N}(t)=\{m: & m \text { is squarefree, } \\
& m \text { has } t \text { prime factors , } \\
& \left.m \text { is composed only of primes } \in Q_{N}\right\} .
\end{aligned}
$$

We put for convenience $M_{N}(0)=\{1\}$.

For any positive integer $t$, we denote by $F(N ; t)$ the number of 
representations of $N$ as the sum of the form $N=p+n$, where $p$ is prime, and $n$ is a positive integer such that $\omega_{N}(n)=t$.

For any positive integer $m$ such that $m \in M_{N}(t)$ with some positive integer $t$, we denote by $G(N ; m)$ the number of representations of $N$ as the sum of the form $N=p+n$, where $p$ is prime, and $n$ is a positive integer such that

$$
\prod_{p \mid n, p \in Q_{N}} p=m
$$

We obviously have

$$
F(N ; t)=\sum_{m \in M_{N}(t)} G(N ; m) .
$$

For any positive integers $t$ and $T$, we put

$$
\begin{aligned}
& \mathscr{P}^{(0)}(N ; t, T)=\sum_{m \in M_{N}(t)} \mathscr{K}^{(0)}(N ; m, T), \\
& \mathscr{K}^{(0)}(N ; m, T)=\sum_{\tau=0}^{2 T}(-1)^{\tau} \mathscr{L}(N ; m, \tau), \\
& \mathscr{C}^{(1)}(N ; t, T)=\sum_{m \in \sum_{N}(t)} \mathscr{K}^{(1)}(N ; m, T) \\
& \mathscr{K}^{(1)}(N ; m, T)=\sum_{\tau=0}^{2 T+1}(-1)^{\tau} \mathscr{L}(N ; m, \tau) \\
& \mathscr{L}(N ; m, \tau)=\sum_{\substack{\mu \in M_{N}(\tau) \\
(\mu, m)=1}} \sum_{\substack{p+n=N \\
m \mu \mid n}} 1
\end{aligned}
$$

LEMMA 2. $\mathscr{H}^{(1)}(N ; t, T) \leqq F(N ; t) \leqq \mathscr{H}^{(0)}(N ; t, T)$.

Proof. We can write

$$
\mathscr{L}(N ; m, \tau)=\sum_{\substack{p+n=N \\
m \mid n}}\left(\begin{array}{c}
\omega_{N}(n)-t \\
\tau
\end{array}\right),
$$

so that

$$
\begin{aligned}
& \mathscr{K}^{(0)}(N ; m, T)=\sum_{\substack{p+n=N \\
m \mid n}} \sum_{\tau=0}^{2 T}(-1)^{\tau}\left(\begin{array}{c}
\omega_{N}(n)-t \\
\tau
\end{array}\right), \\
& \mathscr{K}^{(1)}(N ; m, T)=\sum_{\substack{p+n=N \\
m \mid n}} \sum_{\tau=0}^{2 T+1}(-1)^{\tau}\left(\begin{array}{c}
\omega_{N}(n)-t \\
\tau
\end{array}\right) .
\end{aligned}
$$

Now, since $m \in M_{N}(t)$ and $m \mid n,(2)$ is equivalent to the equality $\omega_{N}(n)=t$. Hence, by (1), we have

$$
\mathscr{K}^{(1)}(N ; m, T) \leqq G(N ; m) \leqq \mathscr{K}^{(0)}(N ; m, T) .
$$


The lemma follows from this and the definitions of $F(N ; t), \mathscr{H}^{(0)}(N ; t, T)$ and $\mathscr{H}^{(1)}(N ; t, T)$.

We further put

$$
\begin{aligned}
& H^{(0)}(N ; t, T)=\sum_{m \in \sum_{N}(t)} K^{(0)}(N ; m, T), \\
& K^{(0)}(N ; m, T)=\sum_{\tau=0}^{2 T}(-1)^{\tau} L(N ; m, \tau), \\
& H^{(1)}(N ; t, T)=\sum_{m \in M_{N}(t)} K^{(1)}(N ; m, T), \\
& K^{(1)}(N ; m, T)=\sum_{\tau=0}^{2 T+1}(-1)^{\tau} L(N ; m, \tau), \\
& L(N ; m, \tau)=\sum_{\substack{\mu \in \sum_{N(\tau)}(\tau) \\
(\mu, m)=1}} \frac{1}{\varphi(m \mu)},
\end{aligned}
$$

where $\varphi(m \mu)$ is Euler's function of $m \mu$.

Lemma 3. Let $T=[5 y(N)]$. Then, as $N \rightarrow \infty$, we have

$$
\begin{aligned}
& H^{(0)}(N ; t, T)=\frac{\{y(N)\}^{t} e^{-y(N)}}{t !}\{1+o(1)\}, \\
& H^{(1)}(N ; t, T)=\frac{\{y(N)\}^{t} e^{-y(N)}}{t !}\{1+o(1)\}
\end{aligned}
$$

uniformly in $t$ with $t<2 y(N)$.

Proof. The formulas in the lemma can be proved quite similarly as Lemma 6 in [6], if we replace the $L(N ; m, \tau)$ 's contained in the definitions of $H^{(0)}(N ; t, T)$ and $H^{(1)}(N ; t, T)$ by

$$
L^{*}(N ; m, \tau)=\sum_{\substack{\mu \in M N_{N}(\tau) \\(\mu, m)=1}} \frac{1}{m \mu \ell} .
$$

Hence it will suffice for the proof of the lemma to show that

$$
L^{*}(N ; m, \tau)=L(N ; m, \tau)\{1+o(1)\}
$$

uniformly in the relevant $L(N ; m, \tau)$ 's.

Now, for each summand of $L(N ; m, \tau)$, the pair of positive integers $m$ and $\mu$ is such that $(m, \mu)=1, m \in M_{N}(t), t<2 y(N), \mu \in M_{N}(\tau), \tau \leqq 10 y(N)+1$, so that, by the definitions of the sets $Q_{N}(t), M_{N}(t)$, and Lemma $1, m \mu$ is squarefree, $\omega(m \mu)<c \log \log N, c>0$, and each of the prime factors of $m \mu$ is greater than $e^{(\log \log N)^{2}}$. Hence 


$$
\begin{aligned}
\frac{1}{m \mu} & <\frac{1}{\varphi(m \mu)}=\frac{1}{m \mu} \prod_{p \mid m \mu}\left(1-\frac{1}{p}\right)^{-1}<\frac{1}{m \mu} \prod_{p \backslash m_{\mu}}\left(1+\frac{2}{p}\right) \\
& <\frac{1}{m \mu}\left(1+2 e^{-(\log 1 \log N)^{2}}\right)^{c \log \log N}=\frac{1+o(1)}{m \mu},
\end{aligned}
$$

or

$$
\frac{1}{m \mu}=\frac{1+o(1)}{\Phi(m \mu)},
$$

from which we see that (3) holds with the required uniformity.

LEMma 4. Let $T$ be an increasing function of $N$ such that $T=$ $O(\log \log N)$. Then, as $N \rightarrow \infty$, we have

$$
\begin{aligned}
& \mathscr{H}^{(0)}(N ; t, T)-H^{0}(N ; t, T) \text { li } N=o\left(\frac{N\{y(N)\}^{t} e^{-y(N)}}{t ! \log N}\right), \\
& \mathscr{C}^{(1)}(N ; t, T)-H^{0}(N ; t, T) \text { li } N=o\left(\frac{N\{y(N)\}^{t} e^{-y(N)}}{t ! \log N}\right)
\end{aligned}
$$

uniformly in $t$ with $t<2 y(N)$, where li $N$ is the logarithmic integral of $N$.

Proof. The definition of $\mathscr{L}(N ; m, \tau)$ can be rewritten as

$$
\mathscr{L}(N ; m, \tau)=\sum_{\substack{\mu \in N_{N}(\tau) \\(\mu, m)=1}} \pi(N ; m \mu, N)
$$

where $\pi(N ; m \mu, N)$ is the number of primes $p$ such that $p<N$ and $p \equiv$ $m \mu(\bmod N)$. Hence, by the definitions of $\mathscr{H}^{(0)}(N ; t, T)$ and $H^{0}(N ; t, T)$, we can write

$$
\begin{aligned}
& \mid \mathscr{C}^{(0)}(N ; t, T)-H^{0}(N ; t, T) \text { li } N \mid \\
& \quad \leqq \sum_{m \in M_{N}(t)} \sum_{\substack{2 T=0 \\
2 T}} \sum_{\substack{\sum_{M N(\tau)}(\tau) \\
(\mu, m)=1}}\left|\pi(N ; m \mu, N)-\frac{\operatorname{li} N}{\Phi(m \mu)}\right| .
\end{aligned}
$$

Put here $m \mu=\nu$, then the same value of $\nu$ occurs at most $d(\nu)$ times, where $d(\nu)$ is the number of divisors of $\nu$; by our assumptions, $\nu$ is squarefree and $\nu \in M_{N}(\tau), \tau<c \log \log N$, so that $\omega(\nu)<c \log \log N$ and

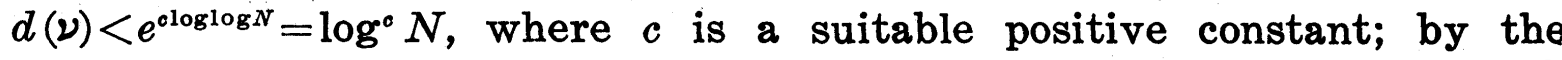
definition of the set $Q_{N}$, each prime factor of $\nu$ is less than $N^{(\log \log N)^{-2}}$, and so $\nu<N^{(\log \log N)^{-1}}$. Hence we have

$$
\left|\mathscr{H}^{(0)}(N ; t, T)-H^{0}(N ; t, T) \operatorname{li} N\right|<\log ^{\circ} N \sum_{(\nu, N)=1}^{N^{c(\log \log N)^{-1}}}\left|\pi(N ; \nu, N)-\frac{\operatorname{li} N}{\varphi(\nu)}\right| .
$$


Now it follows from Bombieri's theorem that

$$
\mathscr{H}^{(0)}(N ; t, T)-H^{0}(N ; t, T) \text { li } N=O\left(N \log ^{-\alpha} N\right)
$$

with arbitrary positive constant $\alpha$. (For our purpose somewhat weaker result than Bombieri's would suffice.) Again, since we assume $t<2 y(N)$,

$$
\frac{\{y(N)\}^{t}}{t !}>\left(\frac{t}{2}\right)^{t} \cdot \frac{1}{t^{t}}=2^{-t}>e^{-2 y(N)} .
$$

Hence we have

$$
\mathscr{C}^{(0)}(N ; t, T)-H^{0}(N ; t, T) \text { li } N=O\left(\frac{N\{y(N)\}^{t} e^{2 y(N)}}{t ! \log ^{\alpha} N}\right) .
$$

Similar result can be obtained for $\mathscr{H}^{(1)}(N ; t, T)$, and, since $y(N) \sim$ $\log \log N$ by Lemma 1 , the lemma follows when we take $\alpha$ sufficiently large.

Lemma 5. Let $T=[5 y(N)]$. Then, as $N \rightarrow \infty$,

$$
\begin{aligned}
& \mathscr{H}^{(0)}(N ; t, T)=\frac{N\{y(N)\}^{t} e^{-y(N)}}{t ! \log N}\{1+o(1)\}, \\
& \mathscr{H}^{(1)}(N ; t, T)=\frac{N\{y(N)\}^{t} e^{-y(N)}}{t ! \log N}\{1+o(1)\}
\end{aligned}
$$

uniformly in $t$ with $t<2 y(N)$.

Proof. The lemma follows from Lemmas 3 and 4.

LEMMA 6. As $N \rightarrow \infty$,

$$
F(N ; t)=\frac{N\{y(N)\}^{t} e^{-y(N)}}{t ! \log N}\{1+o(1)\}
$$

uniformly in $t$ with $t<2 y(N)$.

Proof. The lemma follows from Lemmas 2 and 5.

LEMMA 7. Let $\alpha<\beta$. Let $t$ be a positive integer such that $t=y(N)$ $+u \sqrt{y(N)}$ with $\alpha<u<\beta$. Then, as $N \rightarrow \infty$,

$$
F(N ; t)=\frac{N}{\sqrt{2 \pi y(N)} \log N} e^{-u^{2} / 2}\{1+o(1)\}
$$

uniformly in $t$ with above-mentioned restrictions. 
Proof. This lemma corresponds to Lemma 13 in [6], and can be proved similarly. The Stirling formula plays an important role in the proof.

Lemma 8. Let $\alpha<\beta$, and let $A^{* *}(N ; \alpha, \beta)$ denote the number of representations of $N$ as the sum of the form $N=p+n$, where $p$ is prime, and $n$ is a positive integer such that

$$
y(N)+\alpha \sqrt{y(N)}<\omega_{N}(n)<y(N)+\beta \sqrt{y(N)} .
$$

Then, as $N \rightarrow \infty$, we have

$$
A^{* *}(N ; \alpha, \beta) \sim \frac{N}{\log N} \cdot \frac{1}{\sqrt{2 \pi}} \int_{\alpha}^{\beta} e^{-x^{2} / 2} d x .
$$

Proof. This lemma corresponds to Lemma 14 in [6], and can be proved similarly.

Lemma 9. Let $\alpha<\beta$, and let $A^{*}(N ; \alpha, \beta)$ denote the number of representations of $N$ as the sum of the form $N=p+n$, where $p$ is prime, and $n$ is a positive integer such that

$$
y(N)+\alpha \sqrt{y(N)}<\omega(n)<y(N)+\beta \sqrt{y(N)} .
$$

Then, as $N \rightarrow \infty$, we have

$$
A^{*}(N ; \alpha, \beta) \sim \frac{N}{\log N} \cdot \frac{1}{\sqrt{2 \pi}} \int_{\alpha}^{\beta} e^{-x^{2} / 2} d x .
$$

Proof. We shall estimate the sum

$$
S(N)=\sum_{p<N}\left\{\omega(N-p)-\omega_{N}(N-p)\right\}
$$

in utilizing Brun-Titchmarsh's inequality. For this inequality, cf. Halberstam-Richert [4], p. 110, Mitsui [5], p. 154. Now, noting the fact that a positive integer has at most one prime factor greater than the square root of itself, we argue as

$$
\begin{aligned}
S(N) & =\sum_{p<N} \sum_{\substack{q(\mid N-p) \\
q \notin Q_{N}}} 1=\sum_{p<N} \sum_{\substack{q \backslash, N-p) \\
q \notin Q_{N}, q \leq \sqrt{N}}} 1+O\left(\sum_{p<N} 1\right) \\
& =\sum_{\substack{q \leq \sqrt{N} \\
q \notin Q_{N}}} \sum_{\substack{p<N \\
p \equiv N(\bmod q)}} 1+O\left(\frac{N}{\log N}\right)=\sum_{\substack{q \leq \sqrt{N} \\
q \notin Q_{N}}} \pi(N ; q, N)+O\left(\frac{N}{\log N}\right)
\end{aligned}
$$

where $q$ runs through the primes satisfying the specified conditions. On 
applying Brun-Titchmarsh's inequality to the last sum, we have

$$
\sum_{\substack{q \leq \sqrt{N} \\ q \notin Q_{N}}} \pi(N ; q, N)=O\left(\sum_{\substack{q \leq \sqrt{N} \\ q \notin Q_{N}}} \frac{N}{q \log (N / q)}\right)=O\left(\frac{N}{\log N} \sum_{\substack{q \leq N \bar{N} \\ q \notin Q_{N}}} \frac{1}{q}\right) .
$$

Again, similarly as in the proof of Lemma 4 in [6], we obtain

$$
\sum_{\substack{q \leq \sqrt{N} \\ q \in Q_{N}}} \frac{1}{q}=O(\log \log \log N) .
$$

Thus it has been proved that

$$
S(N)=O\left(\frac{N}{\log N} \log \log \log N\right) .
$$

Now we can prove the lemma similarly as in the proof of Lemma 15 in [6], using this result in the form

$$
\sum_{p+n=N}\left\{\omega(n)-\omega_{N}(n)\right\}=o\left(\frac{N}{\log N} \sqrt{y(N)}\right) .
$$

It follows from this that, for any given $\varepsilon>0$, we can take $N_{1}=N_{1}(\varepsilon)$ so large that, when $N>N_{1}$, the number of representations of $N$ as the sum of the form $N=p+n$ such that the inequality $\omega(n)-\omega_{N}(n)>\varepsilon \sqrt{y(N)}$ holds, is less thau $\varepsilon N / \log N$. Hence, for $N>N_{1}$,

$$
A^{* *}(N ; \alpha, \beta-\varepsilon)-\varepsilon \frac{N}{\log N}<A^{*}(N ; \alpha, \beta)<A^{* *}(N ; \alpha-\varepsilon, \beta)+\varepsilon \frac{N}{\log N} .
$$

From this and Lemma 8, we conclude that

$$
\begin{aligned}
\frac{1}{\sqrt{2 \pi}} \int_{\alpha}^{\beta-\varepsilon} e^{-x^{2} / 2} d x-\varepsilon & \leqq \liminf _{N \rightarrow \infty} \frac{A^{*}(N ; \alpha, \beta) \log N}{N} \\
& \leqq \limsup _{N \rightarrow \infty} \frac{A^{*}(N ; \alpha, \beta) \log N}{N} \leqq \frac{1}{\sqrt{2 \pi}} \int_{\alpha-\varepsilon}^{\beta} e^{-x^{2} / 2} d x+\varepsilon,
\end{aligned}
$$

which gives the lemma.

THE LAST STEP OF THE PROOF OF THE THEOREM. The remaining task is to replace $y(N)$ by $\log \log N$. This can be carried out quite similarly as in the proof of Lemma 16 in [6]. We avoid the repetition. 


\section{References}

[1] E. Bombieri, On the large sieve, Mathematika, 12 (1965), 201-225.

[2] P. X. Gallagher, Bombieri's mean value theorem, Mathematika, 15 (1968), 1-6.

[3] H. HALBERSTAM, On the distribution of additive number-theoretic functions (III), J. London Math. Soc., 31 (1956), 14-27.

[4] H. Halberstam and H.-E. Richert, Sieve Methods, Academic Press, London, 1974.

[5] T. Mrtsur, The Theory of Numbers, Shibundo, Tokyo, 1970 (in Japanese).

[6] M. Tanaka, Some results on additive number theory III, ToKyo J. Math., 2 (1979), 159-182.

Present Addres8:

Department of Mathematics

FACULTY OF SCIENCE

Gakushuin UNIVERSITY

MEJIRO TOSHIMA-KU

TOKYO 171 